\title{
Suzuki-Type of Common Fixed Point Theorems in S-Fuzzy Metric Spaces
}

\author{
M. Jeyaraman and S. Sowndrarajan
}

\begin{abstract}
In this paper, by using of Suzuki-type approach [Suzuki, T., A generalized Banach contraction principle that characterizes metric completeness, Proc. Amer. Math. Soc., 136, 1861-1869, 2008.] we prove new type of Suzuki- type fixed point theorem for non-Archimedean $S$ - fuzzy metric spaces which is generalization of Suzuki-Type fixed point results in $\mathbf{S}$ - metric spaces.
\end{abstract}

Key words — Fixed point, Suzuki-Contraction, S-fuzzy metric space.

\section{INTRODUCTION}

In 1965, the concept of the fuzzy set was initially investigated by Zadeh [13]. Then in 1975, Kramosil and Michalek [4] introduced the fuzzy metric space as a generalization of a metric space. In 1994, George and Veeramani [2] modified the notion of fuzzy metric spaces by using continuous t-norms. Fixed point theorems for contractive mappings in metric spaces have been studied by many authors (see [1], [5], [12]). The Banach contraction principle is the most celebrated fixed point theorem and has been generalized in various directions. In 2008 Suzuki [6] introduced an interesting generalization of Banach contraction principle. Recently, Sedghi, Shobe and Aliouche [8] have defined the concept of S-metric space as a generalization of a metric space, (see [3], [7], [9]-[11]) and proved some fixed point results. In this paper, we introduce the new contractive condition in the frame work of nonArchimedean S-fuzzy metric spaces. We also prove the corresponding coincidence fixed point theorem for two mappings in this framework.

\section{PRELIMINARIES}

\section{A. Definition 2.1}

A binary operation $*:[0,1] \times[0,1] \rightarrow[0,1]$ is a continuous $\mathrm{t}-$ norm if it satisfies the following conditions:

1. $*$ is associative and commutative;

2. $*$ is continuous;

3. $a * 1=a$, for all $\mathrm{a} \in[0,1]$;

4. $a * b \leq c * d$ whenever $a \leq c$ and $b \leq d$, for each $a, b, c, d \in[0,1]$.

Examples for continuous $\mathrm{t}-$ norm are $a \times b=$ $\min \{a, b\}$ and $a \times b=a b$.

Published on July 24, 2021.

M. Jeyaraman, PG and Research Department of Mathematics, Raja Doraisingam Govt. Arts College, Sivagangai, Affiliated to Alagappa University, Karaikudi, India.

(e-mail: jeya.math@gmail.com)

\section{B. Definition 2.2}

A 3 - tuple $(X, S, *)$ is called $S$-fuzzy metric space if $X$ is an arbitrary non - empty set, $*$ is a continuous $\mathrm{t}-$ norm, and $\mathrm{S}$ is a fuzzy set on $\mathrm{X}^{3} \times[0, \infty)$ satisfying the following conditions. For each $x, y, z, a \in X$ and $r, s, t>0$ :

1. $S(x, y, z, t)>0$;

2. $S(x, y, z, t)=1$ if $x=y=z$;

3. $S(x, y, z, t=S(p\{x, y, z\}, t)$, where $p$ is a permutation;

4. $S(x, y, w, r) * S(x, w, z, s) * S(w, y, z, t) \leq$

$S(x, y, z, r+s+t)$, (Tetrahedral inequality).

5. $S(x, y, z,):.(0, \infty) \rightarrow[0,1]$ is continuous.

\section{Example 2.3}

Let $\mathrm{X}=[0,1]$ with a usual metric. Define

$$
S(x, y, z, t)=\min \{M(x, y, t), M(y, z, t), M(z, x, t)\}
$$

where $M(x, y, t)=\frac{t}{t+d(x, y)}$ and $d(x, y)=|x-y|$ for all $x, y \in X$. Then $(\mathrm{X}, \mathrm{S}, *)$ is called $\mathrm{S}$-fuzzy metric space.

\section{Definition 2.4}

A 3 - tuple $(X, S, *)$ is called non-Archimedean S-fuzzy metric space if $\mathrm{X}$ is an arbitrary non - empty set, $*$ is a continuous $\mathrm{t}-$ norm, and $\mathrm{S}$ is a fuzzy set on $\mathrm{X}^{3} \times[0, \infty)$ satisfying the following conditions. For each $x, y, z, w \in X$ and $r, t, u>0$ :

1. $S(x, y, z, t)>0$,

2. $S(x, y, z, t)=1$ if $x=y=z$,

3. $S(x, y, z, t)=S(p\{x, y, z\}, t)$, where $p$ is a permutation

4. $S(x, y, w, r) * S(x, w, z, s) * S(w, y, z, t)$

$$
\leq S(x, y, z, r \vee t \vee u)
$$

where $r \vee t \vee u=\max \{r, t, u\}$, (Tetrahedral inequality). 5. $S(x, y, z,):.(0, \infty) \rightarrow[0,1]$ is continuous.

\section{E. Definition 2.5}

Let $(X, S, *)$ be a $S$ - fuzzy Metric space, $x \in X$ and $\left\{x_{n}\right\}$ be a sequence in $\mathrm{X}$. Then:

1. A sequence $\left\{x_{n}\right\}$ is said to be convergent to $x$ if for every $\varepsilon>0$, there exists $\mathrm{n}_{0} \in \mathbb{N}$ such that $\mathrm{S}\left(x_{n}, x_{n}, x, t\right)>1-\varepsilon$ for all $\mathrm{n} \geq \mathrm{n}_{0}$.

2. A sequence $\left\{x_{n}\right\}$ is said to be a Cauchy sequence if for each $\varepsilon>0$ and there exists $\mathrm{n}_{0} \in \mathbb{N}$ such that $S\left(x_{m}, x_{n}, x_{n}, t\right)>1-\varepsilon$ for all $\mathrm{m}, \mathrm{n} \geq \mathrm{n}_{0}$.

S. Sowndrarajan, Research Scholar, PG and Research Department of Mathematics, Raja Doraisingam Govt. Arts College, Sivagangai, Affiliated to Alagappa University, Karaikudi, Tamil Nadu, India.

(e-mail: sowndariitm ${ }^{\circledR}$ gmail.com) 
3. The S-fuzzy metric space is called complete if every Cauchy sequence is convergent.

\section{F. Lemma 2.6}

Let $(\mathrm{X}, \mathrm{S}, *)$ be an S-fuzzy metric space. If there exists sequence $\left\{\mathrm{x}_{\mathrm{n}}\right\}$ and $\left\{\mathrm{y}_{\mathrm{n}}\right\}$ such that $\lim _{\mathrm{n} \rightarrow \infty} x_{n}=x$ and $\lim _{\mathrm{n} \rightarrow \infty} y_{n}=\mathrm{y}$, then $\lim _{\mathrm{n} \rightarrow \infty} \mathrm{S}\left(x_{n}, x_{n}, y_{n}, \mathrm{t}\right)=S(x, x, y, t)$ for all $x$, $y \in X$.

\section{MAIN RESULTS}

\section{A. Theorem 3.1}

Let $a * b=a b$ for all $\mathrm{a}, \mathrm{b} \in[0,1]$ and $(\mathrm{X}, \mathrm{S}, *)$ be a complete non- Archimedean S - fuzzy metric space. Let T, R: $X \rightarrow X$ be two self maps and $\theta:[0,1) \rightarrow\left(\frac{1}{2}, 1\right]$ be defined by:

$$
\theta(r)=\left\{\begin{array}{c}
1,0 \leq r \leq \frac{\sqrt{5}-1}{2} \\
\frac{1-r}{r^{2}}, \frac{\sqrt{5}-1}{2} \leq r \leq \frac{1}{\sqrt{2}} \\
\frac{1}{1+r}, \frac{1}{\sqrt{2}} \leq r \leq 1
\end{array}\right.
$$

If there exists $r \in[0,1)$ such that for each $x, y, z \in X$, $t>0$ satisfying the condition:

$$
\begin{aligned}
& \max \{S(x, y, T x, t), S(x, y, R x, t)\}^{\theta(r)} \geq S(x, y, z, t) \\
& \quad \text { implies } \\
& \left\{\begin{array}{c}
S(R x, R y, R z, t) * S(T x, T y, T z, t) * \\
S(R x, R y, T z, t) * S(T x, T y, R z, t)
\end{array}\right\} \geq S(x, y, z, t)^{r}
\end{aligned}
$$

Then there exists a unique common fixed point of $\mathrm{T}$ and $\mathrm{R}$.

\section{B. Proof}

At first, we prove that if $v$ is a fixed point of $\mathrm{T}$, then it is also fixed point of $\mathrm{R}$ and vice versa. Hence, let $v$ be a fixed point of $T$, then we will show that $R v=v$.

Taking $x, y=v$ and $z=T v$ in (2) and (3), We get:

$$
1=\max \{S(v, v, T v, t), S(v, v, R v, t)\}^{\theta(r)}>S(v, v, T v, t)
$$

implies

$\left\{\begin{array}{c}S(R v, R v, R T v, t) * S\left(T v, T v, T^{2} v, t\right) \\ * S\left(R v, R v, T^{2} v, t\right) * S(R T v, T v, T v, t)\end{array}\right\} \geq S(v, v, T v, t)^{r}$

$$
=1
$$

Thus, $S(R v, R v, v, t)=1$. i.e., $\mathrm{v}$ is a fixed point of $\mathrm{R}$. Similarly, we can show that if $v$ is a fixed point of $\mathrm{R}$, then it is also fixed point of $\mathrm{T}$. Now to prove our theorem, it is enough to prove that $\mathrm{T}$ and $\mathrm{R}$ has a fixed point.

Putting $x, y=x$ and $z=T x$ in (2) and (3), we get:

$$
\max \{S(x, x, \mathrm{~T} x, t), S(x, x, R x, t)\}^{\theta(r)} \geq S(x, x, T x, t)
$$

implies
$\left\{\begin{array}{c}\mathrm{S}(\mathrm{Rx}, \mathrm{Rx}, \mathrm{RT} x, \mathrm{t}) * \mathrm{~S}\left(\mathrm{Tx}, \mathrm{Tx}, \mathrm{T}^{2} \mathrm{x}, \mathrm{t}\right) * \\ \mathrm{~S}\left(\mathrm{Rx}, \mathrm{Rx}, \mathrm{T}^{2} \mathrm{x}, \mathrm{t}\right) * \mathrm{~S}(\mathrm{RT} \mathrm{x}, \mathrm{Tx}, \mathrm{Tx}, \mathrm{t})\end{array}\right\} \geq \mathrm{S}(\mathrm{x}, \mathrm{x}, \mathrm{Tx}, \mathrm{t})^{\mathrm{r}}$

Hence,

$$
S\left(T x, T x, T^{2} x, t\right) \geq \mathrm{S}(\mathrm{x}, \mathrm{x}, \mathrm{Tx}, \mathrm{t})^{\mathrm{r}} \text { for all } \mathrm{x} \in \mathrm{X}
$$

and

$S(T x, T x, R T x, t) \geq S(x, x, T x, t)$ for all $\mathrm{x} \in \mathrm{X}$.

Putting $x, y=x$ and $z=R x$ in (2.2) and (2.3). Hence, form

$$
\max \{S(x, x, T x, t), S(x, x, R x, t)\}^{\theta(r)} \geq S(x, x, R x, t)
$$

implies

$\left\{\begin{array}{c}S\left(R x, R x, R^{2} x, t\right) * S(T x, T y, T R x, t) * \\ S(R x, R x, T R x, t) * S\left(R^{2} x, T x, T x, t\right)\end{array}\right\} \geq S(x, x, R x, t)^{r}$

Hence,

$S(R x, R x, T R x, t) \geq S(x, x, R x, t)^{r}$ for all $\mathrm{x} \in \mathrm{X}$

Let $\mathrm{x}_{0} \in \mathrm{X}$ be arbitrary and form the sequence $\left\{\mathrm{x}_{\mathrm{n}}\right\}$ by $\mathrm{x}_{1}=$ $\mathrm{Rx}_{0}$ and $x_{2 n+1}=R x_{2 n}, x_{2 n+2}=T x_{2 n+1}$ for $n \in \mathbb{N} \cup\{0\}$. By (6), we have

$$
\begin{aligned}
& S\left(x_{2 n}, x_{2 n}, x_{2 n+1}, t\right)=S\left(T x_{2 n-1}, T x_{2 n-1}, R T x_{2 n-1}, t\right) \\
\geq & S\left(x_{2 n-1}, x_{2 n-1}, T x_{2 n-1}, t\right)^{r} \\
= & S\left(x_{2 n-1}, x_{2 n-1}, x_{2 n}, t\right)^{r} .
\end{aligned}
$$

Also, by (7) we have:

$$
\begin{aligned}
& S\left(x_{2 n+1}, x_{2 n+1}, x_{2 n+2}, t\right)=S\left(R x_{2 n}, R x_{2 n}, T R x_{2 n}, t\right) \\
\geq & S\left(x_{2 n}, x_{2 n}, R x_{2 n}, t\right)^{r} \\
= & S\left(x_{2 n}, x_{2 n}, x_{2 n+1}, t\right)^{r} .
\end{aligned}
$$

By (8), (9) we have:

$S\left(x_{n}, x_{n,}, x_{n+1}, t\right) \geq S\left(x_{n-1}, x_{n-1,}, x_{n}, t\right)^{r}$.

Hence, by induction, we have:

$$
\begin{aligned}
S\left(x_{n}, x_{n,}, x_{n+1}, t\right) & \geq S\left(x_{n_{1},} x_{n_{1},}, x_{n}, t\right)^{r} \\
& \geq \cdots \geq S\left(x_{0}, x_{0}, x_{1}, t\right)^{r^{n}}
\end{aligned}
$$

By Tetrahedral inequality in $\mathrm{S}$ - fuzzy metric space for $\mathrm{m}>n$ we have,

$$
\begin{gathered}
S\left(x_{n,} x_{n,}, x_{m}, t\right) \geq S\left(x_{n,} x_{n,} x_{n+1}, t\right) * S\left(x_{n+1}, x_{n+1}, x_{n+2}, t\right) \\
* \cdots * S\left(x_{m-1}, x_{m-1}, x_{m}, t\right) \\
\geq S\left(x_{0}, x_{0}, x_{1}, t\right)^{r^{n}} * S\left(x_{0}, x_{0}, x_{1}, t\right)^{r^{n+1}}
\end{gathered}
$$




$$
\begin{array}{r}
* \cdots * S\left(x_{0}, x_{0}, x_{1}, t\right)^{r^{m-1}} \\
\geq S\left(x_{0}, x_{0,}, x_{1}, t\right)^{r^{n}} \cdot S\left(x_{0} x_{0}, x_{1}, t\right)^{r^{n+1}} . \\
\cdots . S\left(x_{0}, x_{0}, x_{1}, t\right)^{r^{m-1}}
\end{array}
$$

As $0<r<1$, we have:

$S\left(x_{n}, x_{n,}, x_{m}, t\right)=S\left(x_{0}, x_{0,}, x_{1}, t\right)^{r^{n}\left(1+r+r^{2}+\cdots+r^{m-n-1}\right)}$

$\geq S\left(x_{0}, x_{0}, x_{1}, t\right)^{\frac{r^{m}}{1-r}} \rightarrow 1$ as $\mathrm{m} \rightarrow \infty$.

So, we have $\lim _{n, m \rightarrow \infty} S\left(x_{n}, x_{n}, x_{m}, t\right)=1$.

It follows that $\left\{x_{n}\right\}$ is a Cauchy sequence. Since $X$ is Complete $\mathrm{S}$ - fuzzy metric space, there is some $v$ in $\mathrm{X}$ such that,

$$
\lim _{n \rightarrow \infty} R x_{2 n}=\lim _{n \rightarrow \infty} x_{2 n+1}=v
$$

and

$$
\lim _{n \rightarrow \infty} T x_{2 n+1}=\lim _{n \rightarrow \infty} x_{2 n+2}=v
$$

We show that $v$ is a common fixed point of $\mathrm{T}$ and $\mathrm{R}$.

It is enough to prove that $T v=v$. Now we consider $x \in X$ with $\mathrm{x} \neq \mathrm{v}$.

As $\quad \lim _{n \rightarrow \infty} \mathrm{S}\left(x_{2 n+1}, x_{2 n+1}, T x_{2 n+1}, t\right)=1 \quad$ and $\lim _{n \rightarrow \infty} S\left(x_{2 n+1}, x_{2 n+1}, x, t\right) \neq 1$, therefore there exists some $x_{2 n_{k}+1} \in \mathrm{X}$ such that

$$
\max \left\{\begin{array}{l}
\mathrm{S}\left(x_{2 n_{k}+1}, x_{2 n_{k}+1}, \mathrm{~T} x_{2 n_{k}+1}, \mathrm{t}\right), \\
\mathrm{S}\left(x_{2 n_{k}+1}, x_{2 n_{k}+1}, \mathrm{R} x_{2 n_{k}+1}, \mathrm{t}\right)
\end{array}\right\}^{\theta(\mathrm{r})}
$$

$\geq \mathrm{S}\left(x_{2 n_{k}+1}, x_{2 n_{k}+1}, \mathrm{x}, \mathrm{t}\right)$

implies, $\left\{\begin{array}{c}S\left(R x_{2 n_{k}+1}, R x_{2 n_{k}+1}, R x, t\right) * S\left(T x_{2 n_{k}+1}, T x_{2 n_{k}+1}, T x, t\right) \\ * S\left(R x_{2 n_{k}+1}, R x_{2 n_{k}+1}, T x_{2 n_{k}+1}, t\right) * S\left(R x, T x_{2 n_{k}+1}, T x_{2 n_{k}+1}, t\right) \\ \geq S\left(x_{2 n_{k}+1}, x_{2 n_{k}+1}, x, t\right)^{r}\end{array}\right.$

Hence,

$$
\begin{aligned}
& S\left(x_{2 n_{k}+2}, x_{2 n_{k}+2}, T x, t\right)=S\left(T x_{2 n_{k}+1}, T x_{2 n_{k}+1}, T x, t\right) \\
\geq & S\left(x_{2 n_{k}+1}, x_{2 n_{k}+1}, x, t\right)^{r}
\end{aligned}
$$

Taking limit as $n \rightarrow \infty$, we have:

$$
\begin{gathered}
S(v, v, T x, t)=\lim _{\mathrm{n} \rightarrow \infty} S\left(x_{2 n_{k}+2}, x_{2 n_{k}+2}, T x, t\right) \\
\geq \lim _{\mathrm{n} \rightarrow \infty} S\left(x_{2 n_{k}+1}, x_{2 n_{k}+1}, x, t\right)^{r} \\
=S(v, v, x, t)^{r}
\end{gathered}
$$

Therefore, for each $x \neq v$,

$$
S(v, v, T x, t) \geq S(v, v, x, t)^{r}
$$

Now by induction, we prove that,

$$
S\left(v, v, T^{n} v, t\right) \geq S(v, v, T v, t) \text { for all } n \in \mathbb{N}
$$

For $\mathrm{n}=1$, the inequality is obvious.

Suppose the inequality (13) is true for some $m \in \mathbb{N}$

$$
S\left(v, v, T^{m} v, t\right) \geq S(v, v, T v, t)
$$

Now for $n=m+1$, if $T^{m} v=v$ then

$$
S\left(v, v, T^{m+1} v, t\right)=S(v, v, T v, t)
$$

If $\mathrm{T}^{\mathrm{m}} \mathrm{v} \neq \mathrm{v}$ then by (12)

$S\left(v, v, T^{m+1} v, t\right) \geq S\left(v, v, T^{m} v, t\right)^{r}$

$$
\geq S(v, v, T v, t)^{r}
$$

$>S(v, v, T v, t)$

Hence, the inequality (13) holds for each $n \in \mathbb{N}$.

Let us assume that $\mathrm{Tv} \neq \mathrm{v}$. Now, we prove the following inequality by the principle of mathematical induction $\mathrm{S}\left(T v, T v, T^{n} v, t\right) \geq S(v, v, T v, t)^{r}$ for each $n \in \mathbb{N}(15)$

For $\mathrm{n}=1$, it is obvious. Further from (5) the inequality (15) holds for $n=2$.

Suppose (15) holds for some $\mathrm{n}>2$, then we have:

$S(T v, T v, v, t)=S(v, v, T v, t)$

$$
\begin{gathered}
\geq S\left(v, v, T^{n} v, t\right) * 2 S\left(T v, T v, T^{n} v, t\right) \\
\geq S\left(v, v, T^{n} v, t\right) * 2 S(v, v, T v, t)^{r}
\end{gathered}
$$

Therefore,

$$
S(v, v, T v, t)^{(1-r)} \geq S\left(v, v, T^{n} v, t\right) .
$$

Case I: $0 \leq r \leq \frac{1}{\sqrt{2}}\left(\right.$ hence $\left.\theta(r)=\frac{1-r}{r^{2}}\right)$ Hence, $\int_{\max }\left\{S\left(T^{n} v, T^{n} v, T^{n+1} v, t\right), S\left(T^{n} v, T^{n} v, R T^{n} v, t\right)\right\}^{\theta(r)}$ $\geq S\left(T^{n} v, T^{n} v, T^{n+1} v, t\right)^{\frac{1-r}{r^{2}}}$

$$
\geq S\left(T^{n} v, T^{n} v, T^{n+1} v, t\right)^{\frac{1-r}{r^{2}}}
$$

So, by (16), we have:

$$
\begin{gathered}
\max \left\{S\left(T^{n} v, T^{n} v, T^{n+1} v, t\right), S\left(T^{n} v, T^{n} v, R T^{n} v, t\right)\right\}^{\theta(r)} \\
\geq S\left(T^{n} v, T^{n} v, T^{n+1} v, t\right)^{\frac{1-r}{r^{2}}} \\
\geq S(v, v, T v, t)^{1-r} \\
\geq S\left(v, v, T^{n} v, t\right) \\
=S\left(T^{n} v, T^{n} v, v, t\right)
\end{gathered}
$$


implies that

$$
\begin{gathered}
\left\{\begin{array}{c}
S\left(R T^{n} v, R T^{n} v, R v, t\right) * S\left(T^{n+1} v, T^{n+1} v, T v, t\right) * \\
S\left(R T^{n} v, R T^{n} v, T v, t\right) * S\left(R v, T^{n+1} v, T^{n+1} v, t\right)
\end{array}\right\} \\
\geq S\left(T^{n} v, T^{n} v, v, t\right)^{r}
\end{gathered}
$$

Using (10), we obtain

$S\left(T v, T v, T^{n+1} v, t\right) \geq S\left(v, v, T^{n} v, t\right)^{r}$

$\geq S(v, v, T v, t)^{r}$

So, the inequality (14) holds for eah $n \in \mathbb{N}$. Now $\mathrm{Tv} \neq \mathrm{v}$ and (14) implies that $\mathrm{T}^{\mathrm{n}} \mathrm{v} \neq \mathrm{v}$ (If $T^{n} v=v$, then we find $S\left(T^{n} v, T^{n} v, v, t\right) \geq S(v, v, T v, t)^{r}$ implies:

$S(v, v, T v, t) \leq S(v, v, T v, t)^{r}$

$<S(v, v, T v, t)$, which is not possible.

Hence, (13) implies that:

$$
\begin{gathered}
S\left(v, v, T^{n+1} v, t\right) \geq S\left(v, v, T^{n} v, t\right)^{r} \\
\geq S\left(v, v, T^{n-1} v, t\right)^{r^{2}} \\
\geq S(v, v, T v, t)^{r^{n}}
\end{gathered}
$$

Hence, $\lim _{n \rightarrow \infty} S\left(v, v, T^{n+1} v, t\right)=1$ this implies that $T^{n} \rightarrow v$. From this and (3.1.15), we have:

$$
\begin{aligned}
& S(T v, T v, v, t)=\lim _{n \rightarrow \infty} S\left(T v, T v, T^{n} v, t\right) \\
& \geq \lim _{n \rightarrow \infty} S(v, v, T v, t)^{r} \\
& =S(T v, T v, v, t)^{r} .
\end{aligned}
$$

Thus, $S(T v, T v, v, t)=1$. Which is contrary to our assumption. Hence, $T v=v$. As already proved $v$ is fixed point of $\mathrm{R}$ also. Hence, $T v=R v=v$.

Case II: $\frac{1}{\sqrt{2}} \leq r<1$ (hence $\theta(r)=\frac{1}{1+r}$ ). We will prove that there exist a subsequence $\left\{x_{n_{k}}\right\}$ of $\left\{x_{n}\right\}$ such that:

$$
\begin{aligned}
& S\left(x_{n_{k}}, x_{n_{k}}, T x_{n_{k}}, t\right)^{\frac{1}{1+r}} \geq S\left(x_{n_{k}}, x_{n_{k}}, v, t\right), \\
& \text { or } S\left(x_{n_{k}}, x_{n_{k}}, R x_{n_{k}}, t\right)^{\frac{1}{1+r}} \geq S\left(x_{n_{k}}, x_{n_{k}}, v, t\right),
\end{aligned}
$$

holds for each $k \in \mathbb{N}$. Suppose that for every $n \in \mathbb{N}$. Then by (10) we have,

$$
\begin{aligned}
& \max \left\{S\left(x_{n}, x_{n}, T x_{n}, t\right), S\left(x_{n}, x_{n}, R x_{n}, t\right)\right\}^{\frac{1}{1+r}} \\
& <S\left(x_{n}, x_{n}, v, t\right)
\end{aligned}
$$

Hence,

$S\left(x_{2 n+1}, x_{2 n+1}, T x_{2 n+1}, t\right)^{\frac{1}{1+r}}<S\left(x_{2 n+1}, x_{2 n+1}, v, t\right)$ and $S\left(x_{2 n}, x_{2 n}, R x_{2 n}, t\right)<S\left(x_{2 n}, x_{2 n}, v, t\right)$ holds for every $n \in \mathbb{N}$.

Then by (10) we have:

$$
\begin{gathered}
S\left(x_{2 n+1}, x_{2 n+1}, x_{2 n}, t\right) \\
\geq S\left(x_{2 n+1}, x_{2 n+1}, v, t\right) * S\left(x_{2 n}, x_{2 n}, v, t\right) \\
>S\left(x_{2 n+1}, x_{2 n+1}, T x_{2 n+1}, t\right)^{\frac{1}{1+r}} \\
* S\left(x_{2 n}, x_{2 n}, T x_{2 n}, t\right)^{\frac{1}{1+r}} \\
>S\left(x_{n}, x_{n}, x_{2 n+1}, t\right)^{\frac{r}{1+r}} * S\left(x_{2 n}, x_{2 n}, x_{2 n+1}, t\right)^{\frac{1}{1+r}} \\
=S\left(x_{2 n}, x_{2 n}, x_{2 n+1}, t\right)
\end{gathered}
$$

which is impossible. Hence one of the following holds for each $n$ :

$$
S\left(x_{2 n+1}, x_{2 n+1}, T x_{2 n+1}, t\right)^{\theta(r)} \geq S\left(x_{2 n+1}, x_{2 n+1}, v, t\right)
$$

Or

$$
S\left(x_{2 n}, x_{2 n}, R x_{2 n}, t\right)^{\theta(r)} \geq S\left(x_{2 n}, x_{2 n}, v, t\right)
$$

If the following inequality hold:

$$
S\left(x_{2 n+1}, x_{2 n+1}, T x_{2 n+1}, t\right)^{\theta(r)} \geq S\left(x_{2 n+1}, x_{2 n+1}, v, t\right)
$$

implies that

$$
\begin{aligned}
& \left\{\begin{array}{c}
S\left(R x_{2 n}, R x_{2 n}, R v, t\right) * S\left(T x_{2 n}, T x_{2 n}, T v, t\right) \\
* S\left(R x_{2 n}, R x_{2 n}, T v, t\right) * S\left(R v, T x_{2 n}, T x_{2 n}, t\right)
\end{array}\right\} \\
& \geq S\left(x_{2 n}, x_{2 n}, v, t\right)^{r}
\end{aligned}
$$

Hence,

$S\left(x_{2 n+1}, x_{2 n+1}, T v, t\right)=S\left(R x_{2 n}, R x_{2 n}, T v, t\right)$

$\geq S\left(x_{2 n}, x_{2 n}, v, t\right)^{r}$

Passing to the limit when $n \rightarrow \infty$, we get that $S(v, v, T v, t) \geq 1$, which is possible only if $T v=v$. Similarly, if the following inequality hold.

$$
S\left(x_{2 n}, x_{2 n}, R x_{2 n}, t\right)^{\theta(r)} \geq S\left(x_{2 n}, x_{2 n}, v, t\right)
$$

We have $T v=v$. Thus, we have proved that $v$ is a fixed point of $\mathrm{T}$.

Finally, we prove the uniqueness of the fixed point. Let $v$ and $u$ be to common fixed points of $\mathrm{T}$ and $\mathrm{R}$, such that $v \neq$ $u$. Taking $x, y=v$ and $z=u$ in (2) and (3), we get:

$$
1=\max \{S(v, v, T v, t), S(v, v, R v, t)\}^{\theta(r)} \geq S(v, v, u, t)
$$

implies

$$
\left\{\begin{array}{c}
S(R v, R v, R u, t) * S(T v, T v, T u, t) \\
* S(R v, R v, T u, t) * S(R u, T v, T v, t)
\end{array}\right\} \geq S(v, v, u, t)^{r}
$$

Therefore, $\quad S(v, v, u, t) \geq S(v, v, u, t)^{r}>S(v, v, u, t)$, which is not possible. Hence $v=u$. This completes the proof. Taking $R=T$, we get the Suzuki type result. 


\section{Corollary 3.2}

Let $(X, S, *)$ be a complete $S$-fuzzy metric space. If $T: X \rightarrow$ $X$ be a self mapping and $\theta:[0,1) \rightarrow\left(\frac{1}{2}, 1\right]$ is defined by (1). Assume that there exists $r \in[0,1)$ such that for each $x, y, z \in X$

$$
S(x, y, T x, t)^{\theta(r)} \geq S(x, y, z, t)
$$

implies

$$
S(T x, T y, T z, t) \geq S(x, y, z, t)^{r}
$$

Then $\mathrm{T}$ has a unique fixed point $v \in X$.

\section{Proof}

To prove the corollary, It is enough to set $R=T$ in Theorem (1).

\section{E. Corollary 3.3}

Let $(\mathrm{X}, \mathrm{S}, *)$ be a complete $\mathrm{S}$ - fuzzy metric space. Let $\mathrm{f}, \mathrm{R}$, $\mathrm{T}: \mathrm{X} \rightarrow \mathrm{X}$ be three self-maps and $\theta:[0,1) \rightarrow\left(\frac{1}{2}, 1\right]$ be defined by (2.1). Assume that there exists $r \in[0,1)$ such that for each $x, y, z \in X$,

$$
\max \{S(x, y, f T x, t), S(x, y, f R x, t)\}^{\theta(r)} \geq S(x, y, z, t)
$$

implies

$$
\begin{gathered}
\left\{\begin{array}{c}
S(f R x, f R y, f R z, t) * S(f T x, f T y, f T z, t) * \\
S(f R x, f R y, f T z, t) * S(f R z, f T y, f T x, t)
\end{array}\right\} \\
\geq S(x, y, z, t)^{r}
\end{gathered}
$$

And if $f$ is one-to-one, $f R=R f$ and $f T=T f$, then $f, T$ and $R$ have a unique common fixed point $v \in X$.

\section{F. Proof}

Considering $f R$ and $f T$ as two maps in the given contractive condition of Theorem (3.1), we get a unique common fixed point for $f R$ and $f T$, i.e., $f R v=f T v=v$. Since $f$ is one-to-one, $f R v=f T v$ implies that $R v=T v$. From

$$
\begin{gathered}
1=\max \{S(v, v, f T v, t), S(v, v, f R v, t)\}^{\theta(r)} \\
\geq S(v, v, T v, t)
\end{gathered}
$$

implies

$$
\begin{aligned}
& \left.\begin{array}{c}
S(f R v, f R v, f R T v, t) * S\left(f T v, f T v, f T^{2} v, t\right) \\
* S\left(f R v, f R v, f T^{2} v, t\right) * S(f R T v, f T v, f T v, t)
\end{array}\right\} \\
= & \left\{\begin{array}{c}
S(f R v, f R v, R f T v, t) * S(f T v, f T v, T f T v, t) \\
* S(f R v, f R v, T f T v, t) * S(R f T v, f T v, f T v, t)
\end{array}\right\} \\
= & \left\{\begin{array}{c}
S(v, v, R v, t) * S(v, v, T v, t) * \\
S(v, v, T v, t) * S(R v, v, v, t)
\end{array}\right\} \\
= & S(v, v, T v, t) \\
\geq & S(v, v, T v, t)^{r} .
\end{aligned}
$$

Therefore $T v=v$, hence $v$ is common fixed point of $f, R$ and $T$.

\section{G. Corollary 3.4}

Let $(\mathrm{X}, \mathrm{S}, *)$ be a complete $\mathrm{S}$ - fuzzy metric space. Let $T, R: X \rightarrow X$ be two self maps such that for each $x, y, z \in X$, satisfying the following condition. If there exists $r \in[0,1)$ such that for each $\mathrm{x}, \mathrm{y}, \mathrm{z} \in X$ satisfying the condition

$$
\max \{S(x, y, T x, t), S(x, y, R x, t)\}^{\frac{1}{2}} \geq S(x, y, z, t)
$$

implies

$$
\left\{\begin{array}{c}
S(R x, R y, R z, t) * S(T x, T y, T z, t) \\
* S(R x, R y, T z, t) * S(T x, T y, R z, t)
\end{array}\right\} \geq S(x, y, z, t)^{r}
$$

Then there exists a unique common fixed point of $\mathrm{T}$ and $\mathrm{R}$.

\section{H. Proof}

It is enough to set $r=\frac{1}{2}$ in Theorem 3.1, we get the result.

\section{CONCLUSION}

In this article, we have proved some new type of Suzuki- type fixed point theorem for non-Archimedean S - fuzzy metric spaces which is generalization of Suzuki-Type fixed point results in $\mathrm{S}$ fuzzy metric spaces. Also, we have shown the existence and uniqueness of fixed points for three self maps in the same structure.

\section{REFERENCES}

[1] Aamri.M and Moutawakil D.El, "Some new common fixed poin theorems under strict contractive conditions", J. Math. Anal. Appl., pp. 181-188, Vol. 27, 2002.

[2] George. A. and Veeramani. P., "On some results in fuzzy metric spaces”. Fuzzy sets and systems. pp 395-399, Vol. 64, 1994.

[3] Jong Kyu Kim, Shaban Sedghi, A. Gholidahneh, M. Mahdi Rezaee, "Fixed point theorems in S-metric spaces", East Asian Math. J., Vol. 32 (5), pp. 677-684, 2016.

[4] Kramosil. I and Michalek. J, "Fuzzy metric and statistical spaces", Kybernetica, Vol 11, pp. 336 -344, 1975.

[5] Mihet D., "A Banach contraction theorem in fuzzy metric spaces" Fuzzy Sets Syst, Vol. 144, pp. 431-439, 2004.

[6] Suzuki, T., "A generalized Banach contraction principle that characterizes metric Completeness", Proc. Amer. Math. Soc., Vol. 136 pp. 1861-1869, 2008.

[7] Suzuki, T., "A new type of fixed point theorem in metric spaces", Nonlinear Anal., Vol. 71, pp. 5313-5317, 2009.

[8] Sedghi, S., Shobe, N., Aliouche, A, "A generalization of fixed point theorems in S-metric spaces". Mat. Vesnik Vol 64(3), pp. 258-266 2012.

[9] Shaban Sedghi, I. Altun, N. Shobe, M. A. Salahshour, "Some Properties of S-metric Spaces and Fixed Point Results", Kyungpook Math. J. Vol. 54, pp. 113-122, 2014.

[10] Shaban Sedghi, NV. Dung, "Fixed point theorems on S-metric spaces", Mat. Vesnik, Vol. 66, pp. 113-124, 2014

[11] Shaban Sedghi, Nabi Shobe and Tatjana Dosenovic, "Fixed poin results in S-metric spaces", Nonlinear Funct. Anal. Appl., Vol. 20, pp. 55-67, 2015.

[12] Vasuki, R., Veeramani, P., "Fixed point theorems and Cauchy sequences in fuzzy metric spaces”, Fuzzy Sets Syst, Vol. 13(5), pp. 409$413,2003$.

[13] Zadeh, L. A, "Fuzzy sets", Information and Computation, Vol. 8, pp $338-353,1965$ 


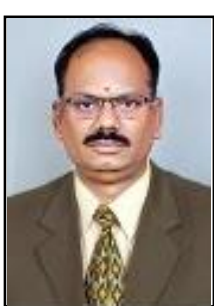

Dr. M. Jeyaraman having more than 20 years of teaching experience and 10 years of research experience, is working as Assistant Professor in PG and Research Department of Mathematics, Raja Doraisingam Government Arts College, Sivagangai, Tamil Nadu in India. He received his Ph.D. degree in 2010. Also, he has M. Phil (Mathematics), M.Ed. He has published several articles in collaboration with profound scholarly professors of various countries and his immense contribution in reputed journals of national and international is more than one hundred twenty five papers. He has presented more than ten research papers in national and international conferences. Five research scholars were awarded $\mathrm{Ph}$. D degree and thirty-six research scholars were awarded an M. Phil degree in Mathematics. He is also reviewer of many prestigious professional bodies like Mathematical Reviews etc. His research interests are Fixed Point Theory, Fuzzy Set Theory, Functional Analysis, Topology and Differential Equations.

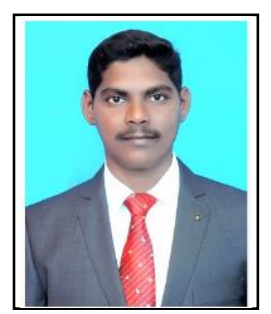

S. Sowndrarajan doing $\mathrm{PhD}$ under the guidance of Dr, M. Jeyaraman at Alagappa University, Karaikudi. He has more than 10 years of teaching experience presently working as Assistant Professor in PG and Research Department of Mathematics, Raja Doraisingam Government Arts College, Sivagangai, Tamil Nadu in India. He received his MSc. degree in Mathematics from IIT Madras and MBA from University of Madras, India. Also, he has Cleared NET, SET and GATE Exams in Mathematics. He has presented 05 research papers in national and international conferences and published more than 10 research articles in reputed international journals. His research interests are Fixed Point Theory, Fuzzy Theory, Functional Analysis. 